Anales de Geografía de la Universidad Complutense ISSN: 0211-9803

http://dx.doi.org/10.5209/AGUC.69333

\title{
La política de energía eólica y sus efectos sobre el desarrollo local. Un análisis a partir del sistema de actores (Loja, Ecuador)
}

\author{
Diana Mendieta Vicuña ${ }^{1}$ J Javier Esparcia Pérez ${ }^{2}$ \\ Recibido: 16 de mayo del 2019 / Enviado a evaluar: 1 de junio del 2019 / Aceptado: 9 de diciembre del 2019
}

Resumen. La política energética ecuatoriana pretende que las centrales eléctricas contribuyan al desarrollo local en las áreas en las que se implantan. A partir del caso de estudio del Parque Eólico Villonaco (PEV), la pregunta de investigación que se plantea es si el Plan de Desarrollo Territorial Comunitario (PDTC) que se está implementando en la zona de influencia del Parque, está teniendo efectos socio-territoriales positivos. Se utiliza una metodología cualitativa, a partir de entrevistas semiestructuradas a actores clave. Los resultados obtenidos indican que la central eólica ha empezado a tener efectos positivos en el ámbito de los equipamientos educativos, la infraestructura vial, o en el alumbrado público. Sin embargo, aun no se evidencia un impacto significativo en cuanto al empleo y a la dinamización de la economía local. En este sentido, los principales efectos multiplicadores se han localizado fuera del área de influencia directa de la Central.

Palabras clave: Energías renovables; desarrollo local; sistema de actores.

\section{[en] Wind energy policy and its effects on local development. An analysis from the stakeholder system (Loja, Ecuador)}

\begin{abstract}
Ecuador's energy policy aims for power plants to contribute to local development in the areas in which they are implemented. Based on the Villonaco Wind Farm (PEV) case study, the research question is whether the Community Territorial Development Plan (PDTC) being implemented in the Park's area of influence, is having positive socio-territorial effects. A qualitative methodology is used, based on semi-structured interviews with stakeholders. The results obtained indicate that the wind power plant has begun to have positive effects in the field of educational equipment, road infrastructure, or public lighting. However, a significant impact on employment and the dynamization of the local economy is still not evident. In this sense, the main multiplier effects have been located outside the direct area of influence of the power plant.
\end{abstract}

Key words: Renewable energies; local development; stakeholder analysis.

Instituto de Desarrollo Local. Universidad de Valencia

E-mail: ga.diylab@gmail.com

2 Instituto de Desarrollo Local. Departamento de Geografía-Universidad de Valencia.

E-mail: javier.esparcia@uv.es 
[fr] La politique éolienne et ses effets sur le développement local. Une analyse basée sur le système des acteurs (Loja, Equateur)

Résumé. La politique énergétique de l'Équateur vise à ce que les centrales électriques contribuent au développement local dans les régions où elles sont mises en œuvre. Sur la base de l'étude de cas du parc éolien de Villonaco (PEV), la question de recherche est de savoir si le plan de développement territorial (PDTC) mis en œuvre dans la zone d'influence du parc a des effets socio-territoriaux positifs. Une méthodologie qualitative est utilisée, basée sur des entretiens semi-structurés avec les parties prenantes. Les résultats obtenus indiquent que la centrale éolienne a commencé à avoir des effets positifs dans le domaine des équipements éducatifs, des infrastructures routières ou de l'éclairage public. Cependant, un impact significatif sur l'emploi et la dynamisation de l'économie locale ne sont toujours pas évidents. En ce sens, les principaux effets multiplicateurs ont été localisés en dehors de la zone d'influence directe de la centrale.

Mots clés: Énergies renouvelables; développement local; système des acteurs.

Cómo citar. Mendieta Vicuña, D. y Esparcia Pérez, J. (2020): La política de energía eólica y sus efectos sobre el desarrollo local. Un análisis a partir del sistema de actores (Loja, Ecuador). Anales de Geografía de la Universidad Complutense, 40(1), 73-95.

Sumario. 1. Introducción. 2. Caso de estudio, objetivos, metodología y fuentes. 3. Efectos del Parque Eólico Villonaco sobre la calidad de vida, empleo y tejido productivo local. 3.1. Efectos sobre la calidad de vida de la población: educación, infraestructuras viales y saneamiento, y electrificación. 3.2. Efectos sobre la creación de empleo. 3.3. Efectos sobre las actividades económicas del territorio. 4. Discusión. 5. Conclusiones. 6. Bibliografía.

\section{Introducción}

Con frecuencia las zonas rurales quedan olvidadas por las administraciones públicas, o al menos insuficientemente atendidas, especialmente las zonas rurales pobres, desfavorecidas o más alejadas de los centros urbanos. Es innegable que el papel de muchas de estas áreas suele ser el de suministro de materias primas, alimentos, agua o energía (además de mano de obra). Hace ya años Dos Santos (2003) planteaba que las relaciones entre países ricos y pobres eran básicamente de explotación; en esa línea, la teoría de la dependencia y de centro-periferia ha sido la base de la interpretación de un marco de relaciones desiguales y dependencia funcional (Hidalgo, 2012), que se ha trasladado a regiones y áreas más (áreas urbanas) y menos desarrolladas (áreas rurales). Tanto estos como los enfoques neoliberales posteriores han sido superados, al menos conceptualmente, a la hora de referirse al desarrollo de regiones en desarrollo, incluidas áreas rurales pobres. Así, desde finales de los años 80 (coincidiendo con el Informe Bruntland, en 1987, y la Cumbre de Río, en 1992) se plantea un marco conceptual en el que esa disponibilidad de recursos constituye la base de estrategias de desarrollo local sostenible. Cómo desde las políticas públicas se enfoque la utilización de los recursos locales resulta fundamental para la supervivencia de muchas áreas rurales. Baste recordar que, a escala mundial, la mayor proporción de trabajadores pobres depende del medio rural (OIT, 2015). Por tanto, los recursos endógenos constituyen una parte fundamental en el fomento de estrategias o 
procesos de desarrollo local, atendiendo con ello a la sostenibilidad social, económica y ambiental de los territorios en los que se aplican (Alburquerque, 2017).

La utilización de recursos endógenos, como los energéticos renovables, tiene una estrecha relación con las políticas de desarrollo local en áreas rurales (también denominado desarrollo territorial), en tanto que su aprovechamiento puede suponer una contribución a la sostenibilidad del territorio (Tribunal de Cuentas Europeo, 2018). En este sentido, la ONU reconoce la importancia de la energía sostenible para erradicar la pobreza y abordar el cambio climático (ONU, 2010; SEFORALL, 2019). En particular, para garantizar varios de los Objetivos de Desarrollo Sostenible (como el acceso a una "Energía asequible y no contaminante"), recomienda una mayor participación de las energías renovables en el mix energético (ONU, 2015). La energía (renovable) es fundamental para la producción de alimentos, aumento de ingresos o empleo (Moreno y López, 2008). Sin embargo, las instalaciones de energía eólica también pueden tener efectos negativos en su entorno (Burrows, 2018), si bien estos tienden a ser menores respecto de energías no renovables (Markandya, 2012).

Está en todo caso demostrado que estas instalaciones pueden favorecer procesos de desarrollo local (Observatorio Europeo Leader, 1999; Walker y Devine-Wright, 2008; Faulín et al., 2009), especialmente cuando se asocian con determinadas condiciones preexistentes en el territorio, como la disponibilidad de recursos endógenos (tanto físicos como socio-económicos) (Burguillo y Del Río, 2008), un cierto tejido empresarial y productivo (oferta de equipos tecnológicos y servicios especializados), o un grupo amplio de beneficiarios directos o indirectos. Además, pueden contribuir al nacimiento de nuevas actividades económicas (Burguillo y Del Río, 2008; IEA-RETD-TCP, 2016) y al fortalecimiento de las existentes (demanda de bienes y servicios desde las propias centrales).

Estas centrales no siempre garantizan el desarrollo económico, ni una distribución equitativa de la renta generada (especialmente en países en desarrollo). Así ocurre, por ejemplo, cuando el empleo local que se crea es temporal y de baja cualificación (Munday et al., 2011), o cuando están ausentes las condiciones señaladas anteriormente (ADAS, 2003). En estos casos, las políticas de desarrollo local (Magnani, 2012; Slee, 2015), determinadas políticas de compensación pueden contrarrestar las limitaciones existentes, contribuyendo a crear oportunidades de desarrollo local en las zonas de próximas (Burguillo y Del Río, 2008; IRENA, 2017).

En Ecuador, la planificación nacional (Plan Nacional para el Buen Vivir) plantea la construcción de nuevas centrales de generación eléctrica renovable (SENPLADES, 2009; 2013), como vía de diversificación y fortalecimiento del sector energético. Paralelamente, en el marco de esas políticas de compensación, el Ministerio de Electricidad y Energía Renovable (MEER) plantea que estas nuevas centrales, además de aprovechar los recursos naturales, contribuyan al desarrollo de sus zonas de influencia (Poveda et al., 2017).

Así, en la fase de construcción, las empresas generadoras han de implementar planes de desarrollo local en las zonas de influencia. Estos incluyen obras de infraestructura y servicios públicos (carreteras y caminos, de agua potable, alcantarillado, infraestructura educativa, sanitaria, recreativa, etc.), actuaciones 
ambientales y proyectos de desarrollo productivo. Por su parte, en la fase de operación comercial se busca este mismo objetivo a través de la aplicación de la Ley Orgánica del Servicio Público de Energía Eléctrica-LOSPEE (2015), que establece (Art. 56) que, para las generadoras de electricidad a cargo de empresas públicas, el 30 $\%$ del beneficio obtenido por la venta de energía debe destinarse a proyectos de desarrollo local en su área de influencia.

\section{Caso de estudio, objetivos, metodología y fuentes}

En este contexto, se plantea un caso de estudio, el Parque Eólico Villonaco (PEV, de solo $16,5 \mathrm{MW}$ ) y su zona de influencia, comprendida por diez barrios periurbanos de los municipios de Loja y Catamayo (Provincia de Loja, Ecuador), situados a una distancia aproximada de entre 0,4 y 4,5 km desde los aerogeneradores (Figura 1). El interés radica, en primer lugar, en que es el primer proyecto eólico en el país, y ya es posible llevar a cabo una aproximación a como influye en el desarrollo de su zona de influencia. En segundo lugar, porque en este territorio la Corporación Eléctrica del Ecuador $^{3}$ - Unidad de Negocio GENSUR ${ }^{4}$ (CELEC EP GENSUR) implementa un Plan de Desarrollo Territorial Comunitario (PDTC), como resultado de la política del MEER. Y, en tercer lugar, porque a diferencia de otros proyectos de energías renovables, el PEV contó inicialmente con la plena aceptación de la comunidad local.

Pese a la importante influencia que recibe de la ciudad de Loja (a tan solo $10 \mathrm{~km}$.), la zona de estudio ${ }^{5}$ responde a características típicas del medio rural. El carácter montañoso del paisaje se modifica gradualmente en dirección a Loja, en una sucesión de áreas agrícolas y ganaderas y otras sin ningún uso aparente (debido al progresivo abandono de la agricultura). El sistema de asentamientos se caracteriza por caseríos dispersos en un relieve irregular. Hay déficits característicos, como el escaso o nulo suministro de agua potable y de electricidad (alumbrado público) la insuficiente o inexistente infraestructura de alcantarillado (GAD Municipal de Loja, 2011).

La hipótesis de investigación de este trabajo es que, en tanto que política de compensación, la inversión que la legislación ecuatoriana prevé para el desarrollo local en la zona de influencia del PEV (tanto a través del PDTC como de posibles efectos directos), está obteniendo los resultados previstos, contribuyendo a generar actividades económicas y, en general, mejorar la calidad de vida de la población. Si bien no ha transcurrido un plazo aún amplio desde la puesta en marcha del PEV $^{6}$

\footnotetext{
${ }^{3}$ Empresa pública de generación, transmisión, distribución y comercialización de energía eléctrica.

${ }_{5}^{4}$ Empresa creada en el año 2011. Opera el PEV.

${ }_{6}^{5}$ Corresponde a la zona de influencia del PEV, definida por CELEC EP GENSUR.

${ }^{6}$ En este trabajo se utiliza indistintamente los términos parque eólico y central eólica para hacer referencia al conjunto de infraestructuras, unidades de generación y demás equipos instalados para la generación de electricidad en el PEV.
} 
(2013), sí es posible valorar ya los primeros efectos socio-territoriales del PDTC y, en general, del PEV. Para responder a esta hipótesis se plantea como objetivo central el análisis de las expectativas y la valoración de la población local y otros actores sobre la capacidad del PEV y del PDTC de generar efectos positivos en su zona de influencia, en tres ámbitos: la calidad de vida de la población local (en particular en educación, infraestructura vial, transporte público y electrificación), el empleo (directo e indirecto) y, finalmente, el tejido productivo local.

Figura 1. Ubicación del Parque Eólico Villonaco y su zona de influencia (Loja, Ecuador).

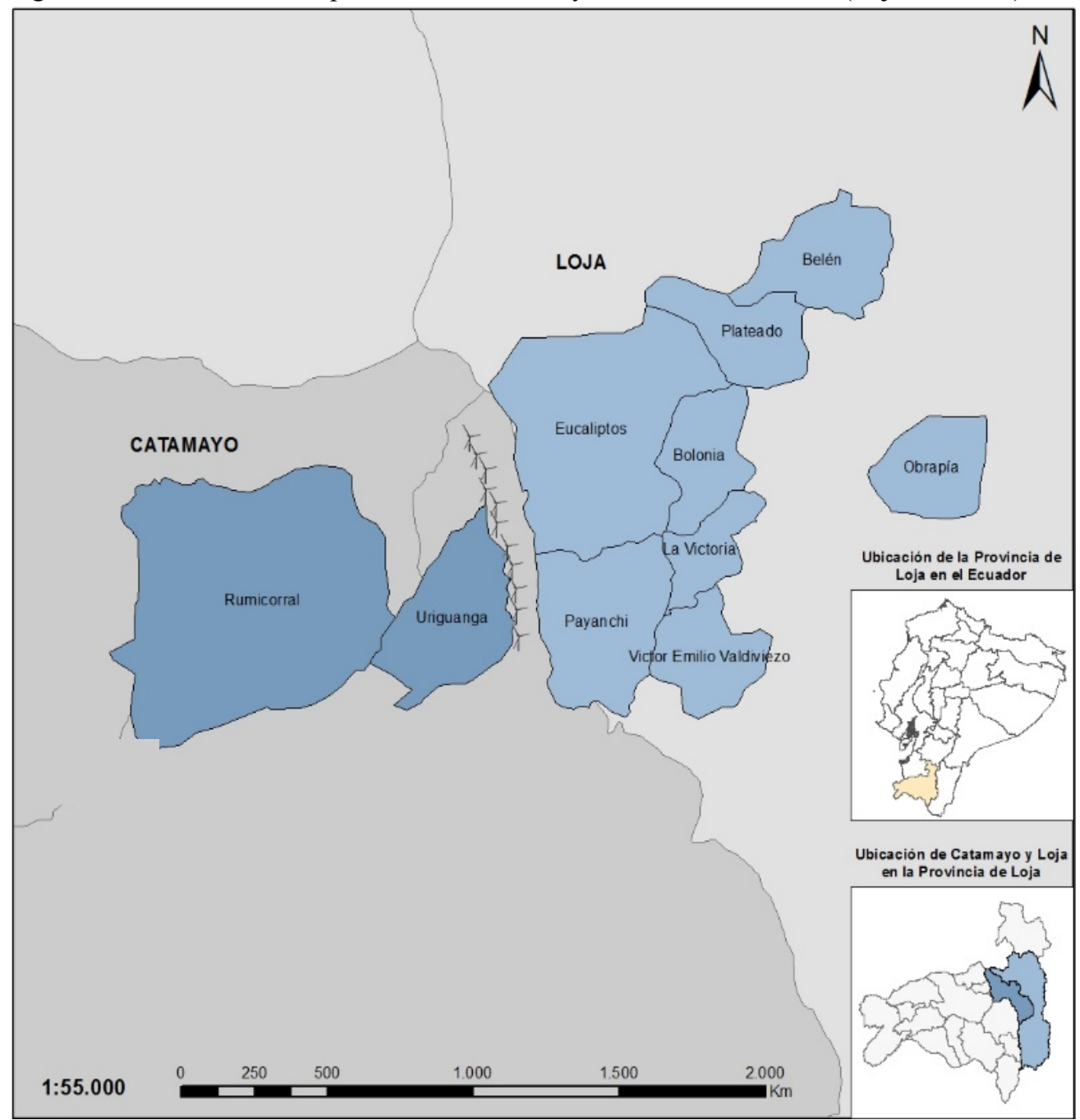

Fuente: CELEC EP GENSUR. Elaboración propia. 
El análisis se aplica a tres ámbitos: la calidad de vida de la población local (en particular en educación, infraestructura vial, transporte público y electrificación), el empleo (directo e indirecto) y, finalmente, el tejido productivo local.

Desde el punto de vista metodológico, se analizan las expectativas y valoración de los diferentes actores porque son los que están 'dentro'de la realidad estudiada (Kvale, 2011) y, por tanto, poseen una información y visión precisa (difícil de obtener por otros medios) respecto de la realidad social local (Ruiz Ruiz, 2009). Por ello la fuente de información primaria principal son entrevistas semiestructuradas (recogidas entre diciembre de 2014 y marzo de 2015), a una muestra de 32 actores, que incluye a aquellos involucrados directamente en la implementación del PDTC (solo dos de los que hubiesen debido estar, no pudieron ser entrevistados).

El guion de la entrevista se ha diseñado en torno a temáticas clave y se ha estructurado en dos secciones. La primera, sobre los procesos de desarrollo local vinculados con la energía eólica, con el objetivo de recoger la valoración y percepción respecto de los efectos directos (positivos o negativos) de la presencia del PEV en el territorio. Por su parte, la segunda sección se dedica a la valoración acerca de las actuaciones del PDTC, es decir, el potencial, limitaciones y beneficios que éste tiene para la comunidad local.

El proceso de análisis cualitativo se inicia con la agregación de la información primaria a partir de la formulación de tipologías, lo que permite obtener una imagen estructurada de los discursos de los actores. Las tipologías se definen a partir del guion de la entrevista, resultando en ocho grandes categorías (códigos) y setenta y cuatro subcategorías (subcódigos), base para el análisis e interpretación de la información (Kvale, 2011). La información codificada permite un análisis sistemático de las percepciones y valoraciones de los actores a partir de la frecuencia de aparición de las distintas temáticas en el discurso de cada actor, o la cantidad de actores que abordan cada tema ${ }^{7}$. Este proceso permite, por un lado, valorar la importancia que los entrevistados otorgan a los diferentes temas y, por otro, identificar y analizar las relaciones significativas entre las temáticas y elementos presentes en sus discursos (Creswell, 2014) ${ }^{8}$ y los procesos y fenómenos estudiados (Figura 2). El proceso metodológico de análisis de la información se ha llevado a cabo a través de MAXQDA, software para análisis cualitativo de información.

\footnotetext{
${ }^{7}$ Estos procedimientos metodológicos han sido previamente desarrollados y analizados con detalle en Mendieta, D. y Esparcia, J.

${ }^{8}$ Un análisis diferente es el de las relaciones entre los actores, o de las estrategias de poder que éstos despliegan en el territorio.
} 
Figura 2. Proceso de organización, tratamiento y análisis de la información.

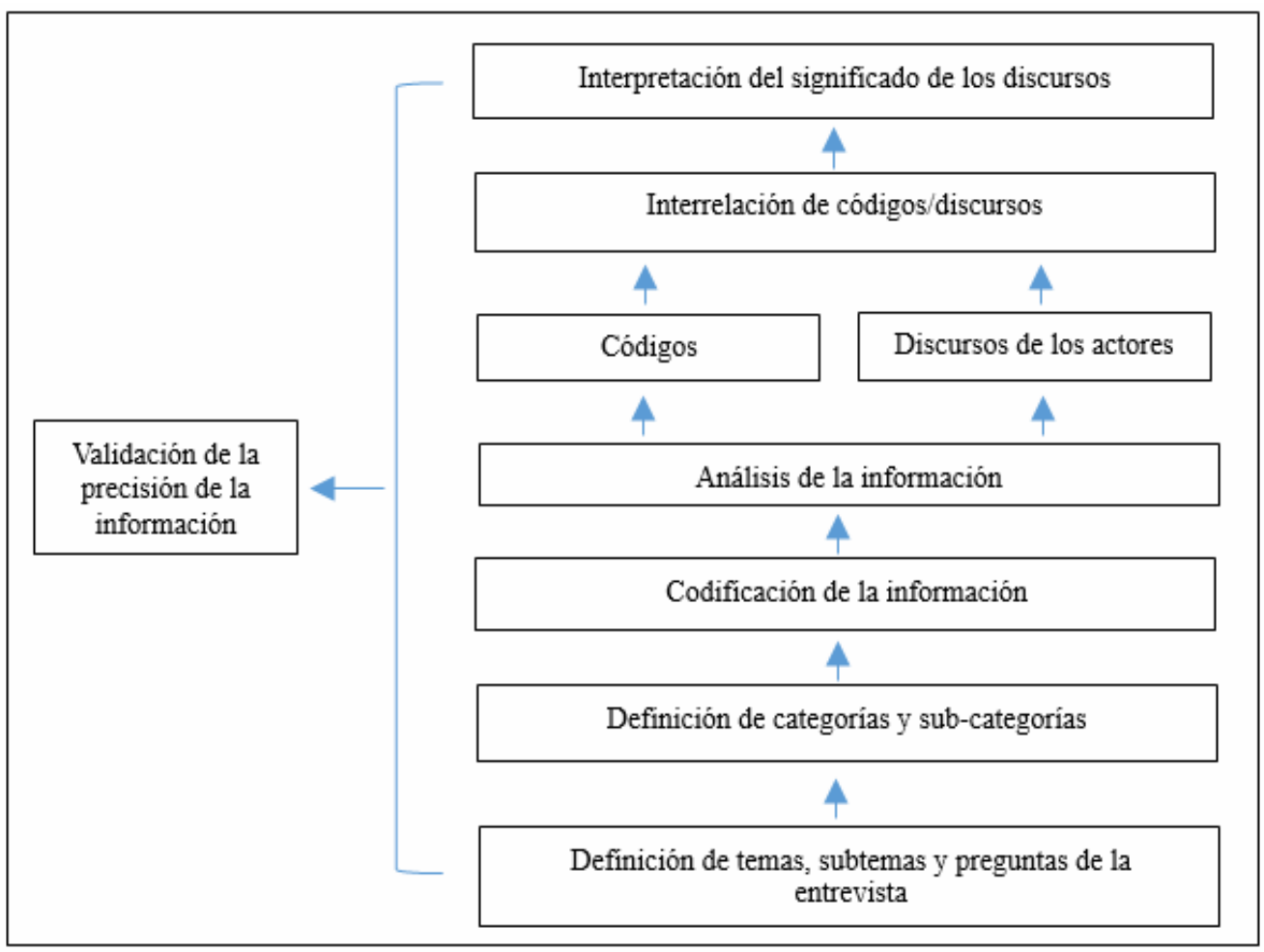

Fuente: Elaborado a partir de Creswell, 2014: 197 (Mendieta, D. y Esparcia, J., 2018)

Se han identificado dos grupos de actores, los de carácter institucional y los beneficiarios potenciales (Cuadro 1). A su vez, los primeros pueden subdividirse en tres subgrupos, según su papel en el territorio: diseño de la política energética, financiación de las actuaciones y ejecución de obras. Por su parte, los segundos se dividen entre actores de carácter social (representan a la población potencialmente beneficiada por las actuaciones del PDTC) y los actores económicos (entre los que se encuentran tanto los proveedores locales de bienes y servicios para la central, como los beneficiarios de actuaciones orientadas al desarrollo productivo, caso de los agricultores). 
Cuadro 1. Tipología de actores locales, porcentaje y número de entrevistados.

\begin{tabular}{|c|c|c|c|}
\hline & \\
\hline & & & $\%\left(n^{\circ}\right.$ actores $)$ \\
\hline \multirow{5}{*}{ 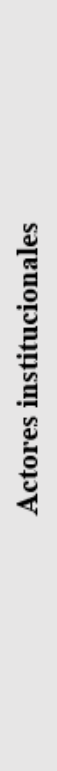 } & $\begin{array}{l}\text { Política } \\
\text { energética }\end{array}$ & $\begin{array}{l}\text { Ministerio de Electricidad y Energía Renovable } \\
\text { (Personal directivo en energía renovable y personal técnico } \\
\text { de desarrollo territorial) }\end{array}$ & $6 \%(2)$ \\
\hline & $\begin{array}{l}\text { Financiación } \\
\text { de actuaciones }\end{array}$ & CELEC EP GENSUR (Jefe de la Central Eólica) & $3 \%(1)$ \\
\hline & \multirow[b]{3}{*}{$\begin{array}{l}\text { Ejecución de } \\
\text { obras }\end{array}$} & CELEC EP GENSUR (Téenico de desarrollo territorial) & $3 \%(1)$ \\
\hline & & $\begin{array}{l}\text { Gobiernos Autónomos Descentralizados municipales de Loja } \\
\text { y Catamayo } \\
\text { (Alcaldesa, Alcalde o su delegado) }\end{array}$ & $6 \%(2)$ \\
\hline & & $\begin{array}{l}\text { Entidades públicas del gobierno nacional con jurisdicción } \\
\text { zonal, provincial, distrital: Coordinación Distrital de } \\
\text { Educación; Dirección Provincial del Ministerio de } \\
\text { Transporte y Obras Públicas; Dirección Provincial del } \\
\text { Ministerio de Agricultura, Ganadería, Silvicultura y Pesca; } \\
\text { Dirección Provincial de la Agencia de Aseguramiento del a } \\
\text { Calidad del Agro; Empresa Eléctrica Regional del Sur } \\
\text { (Técnicos) }\end{array}$ & $19 \%(6)$ \\
\hline \multirow{5}{*}{ ضั } & \multirow{4}{*}{ Actores sociales } & $\begin{array}{l}\text { Presidentes de barrios (comités pro-mejoras y juntas de } \\
\text { desarrollo barrial) }\end{array}$ & \multirow{4}{*}{$47 \%(15)$} \\
\hline & & Presidentes de dos comités de gestión del agua de la zona & \\
\hline & & Directoras de centros educativos beneficiados & \\
\hline & & $\begin{array}{l}\text { Presidenta del Comité de madres y padres de familia de uno } \\
\text { de los centros educativos }\end{array}$ & \\
\hline & $\begin{array}{l}\text { Actores } \\
\text { económicos }\end{array}$ & $\begin{array}{l}\text { Asociación Virgen de Guadalupe (prestación de servicios de } \\
\text { limpieza y jardinería), Asociación de Agricultores Villonaco, } \\
\text { pequeños agricultores locales }\end{array}$ & $16 \%(5)$ \\
\hline \multicolumn{2}{|c|}{ Total } & & $100 \%(32)$ \\
\hline
\end{tabular}

Fuente: Elaborado a partir de las entrevistas realizadas a los representantes de CELEC EP GENSUR.

Adicionalmente, se han utilizado otras fuentes de información cualitativa, como la observación directa (vías de comunicación, escuelas, infraestructura eléctrica, etc.) y las correspondientes notas de campo, fotografías, etc., así como fuentes secundarias provenientes de organismos oficiales (páginas web, planes de desarrollo y ordenamiento territorial, informes de rendición de cuentas, etc.), y de medios de comunicación local y nacional. 


\section{Efectos del Parque Eólico Villonaco sobre la calidad de vida, empleo y tejido productivo local}

Se diferencian dos grandes tipos de inversiones, las correspondientes al PEV (construcción, instalación, operación, etc.) y las del PDTC. Este último tiene un doble objetivo, la compensación por los impactos derivados de su construcción (el Plan se inicia en junio de 2012, antes de su operación comercial) y la promoción del desarrollo local en la zona de influencia. El objetivo último es mejorar las condiciones de vida de la población local, principalmente a través de la ejecución de obras de infraestructura, en el marco del Plan Nacional para el Buen Vivir -PNBV(SENPLADES, 2009, 2013). De este plan, el PDTC asume otros objetivos, como auspiciar la igualdad, cohesión e integración social y territorial en la diversidad; incrementar las capacidades y potencialidades de la ciudadanía; mejorar la calidad de vida de la población; e impulsar la transformación de la matriz productiva.

Cuadro 2. Actuaciones, beneficiarios e inversión del Plan de Desarrollo Territorial Comunitario.

\begin{tabular}{|c|c|c|c|}
\hline Ámbito & Actuaciones & Beneficiarios & Inversión \\
\hline $\begin{array}{l}\text { Infraestructura } \\
\text { escolar } \\
(2013-2014)\end{array}$ & $\begin{array}{l}\text { 1. Adecuación y rehabilitación de aulas, } \\
\text { espacios deportivos y baterías sanitarias } \\
\text { 2. Construcción de aula y fosa séptica } \\
\text { 3. Dotación de equipo informático, } \\
\text { mobiliario y juegos recreativos } \\
\text { 4. Reparaciones de infraestructura física } \\
\text { 5. Conservación de áreas verdes }\end{array}$ & $\begin{array}{l}\text { a) } 559 \text { estudiantes } \\
\text { b) } 4 \text { Barrios (Obrapía, } \\
\text { Tierras Coloradas, } \\
\text { Payanchi, Rumicorral) } \\
\text { c) } 5 \text { centros educativos } \\
\text { (de un total de } 7 \text { ) }\end{array}$ & $280.561 \mathrm{USD}^{\mathrm{a}}$ \\
\hline \multirow{2}{*}{$\begin{array}{l}\text { Infraestructura } \\
\text { vial y } \\
\text { alcantarillado } \\
\text { (abr. 2013 - dic. } \\
\text { 2013) }\end{array}$} & $\begin{array}{l}\text { 1. Construcción del acceso a la Subestación } \\
\text { Villonaco }(5,5 \mathrm{~km} \text {.) } \\
\text { 2. Construcción y reparación de las redes de } \\
\text { alcantarillado sanitario adyacentes a la vía }\end{array}$ & $\begin{array}{l}\text { a) } 1055 \text { habitantes } \\
\text { b) } 3 \text { Barrios } \\
\text { (Eucaliptos, Bolonia y } \\
\text { Plateado) }\end{array}$ & 2.624.621 USD ${ }^{\mathrm{b}}$ \\
\hline & $\begin{array}{l}\text { 3. Rehabilitación de la vía Loja - Catamayo, } \\
\text { accesos y caminos internos del PEV ( } 17 \\
\mathrm{~km} \text {.) }\end{array}$ & $\begin{array}{l}\text { a) } 344 \text { habitantes } \\
\text { b) } 4 \text { Barrios (Tierras } \\
\text { Coloradas, Payanchi, } \\
\text { Uriguanga y Rumicorral) }\end{array}$ & $909.565 \mathrm{USD}^{\mathrm{c}}$ \\
\hline $\begin{array}{l}\text { Obras de } \\
\text { electrificación } \\
\text { (jul. } 2012 \text { - dic. } \\
\text { 2012) }\end{array}$ & $\begin{array}{l}\text { 1. Montaje de luminarias de alumbrado } \\
\text { público } \\
\text { 2. Extensión de red en media y baja tensión } \\
\text { 3. Instalación de transformadores }\end{array}$ & $\begin{array}{l}6 \text { Barrios (Tierras } \\
\text { Coloradas, Uriguanga, } \\
\text { Eucaliptos, Obrapía, } \\
\text { Payanchi, Rumicorral) }\end{array}$ & 35.569 USD \\
\hline
\end{tabular}

\footnotetext{
${ }^{a}$ Inversión realizada por CELEC EP GENSUR

${ }^{\mathrm{b}}$ Inversión compartida: 90\% CELEC EP GENSUR, $10 \%$ GAD Municipal de Loja

${ }^{\mathrm{c}}$ Inversión compartida: 61 \% CELEC EP GENSUR, 39 \% Ministerio de Transporte y Obras Públicas (MTOP)

Fuente: Elaborado a partir de CELEC EP GENSUR (2019) y Acuerdos de Cooperación Interinstitucional entre CELEC y EERSSA -Empresa pública regional de distribución y comercialización de electricidad(Junio 2012), MTOP (Febrero 2013), GAD Municipal Loja (Abril 2013) y Ministerio de Educación (Junio 2013).
} 
El PDTC, en cuya elaboración ha participado la población local, prioriza la atención a necesidades básicas a través de 11 áreas de intervención: educación, infraestructura viaria, electrificación, infraestructura comunitaria, agua potable, alcantarillado y saneamiento, desarrollo económico, salud, seguridad ciudadana, transporte público, y servicio telefónico. En la fecha de realización del trabajo de campo se habían llevado a cabo actuaciones en educación, infraestructura viaria y desarrollo económico (Cuadro 2), mediante acuerdos de colaboración interinstitucional (dado que la empresa o no tiene competencias legales o no tiene capacidad técnica).

\subsection{Efectos sobre la calidad de vida de la población: educación, infraestructuras viales y saneamiento, y electrificación}

En primer lugar, respecto de las infraestructuras y servicios públicos educativos en estas áreas rurales, la situación previa se caracterizaba por deficiencias de funcionalidad, seguridad e higiene, entre otras, frente a la significativa mejor situación de los centros educativos urbanos (con mayor prestigio y visibilidad social). La falta de soluciones ya había llevado a la comunidad local a iniciar acciones de mejora con sus propios recursos y trabajo colectivo. No obstante, ha sido el PDTC el instrumento que ha permitido concluir diversas de estas obras, así como acometer nuevas actuaciones en otros cuatro centros educativos (Cuadro 2).

Los actores entrevistados valoran muy positivamente estas intervenciones (Figuras 3 y 4), por sus efectos sobre la calidad educativa. Destacan mejoras en las condiciones ambientales (mayor luminosidad, mejor ventilación, menor ruido, etc.), en la funcionalidad y calidad de los servicios (mayor espacio y menor hacinamiento, mayor comodidad y funcionalidad, nuevos servicios higiénicos), en la seguridad (vallas, muros, etc., que protegen a los estudiantes de riesgo de atropellos, o permiten un mayor control de las personas ajenas al centro), en el mayor acceso a las TIC (instalación de salas de informática) o en el fomento del deporte (equipamientos deportivos). Todo ello repercute directamente en el proceso de aprendizaje y en el desarrollo y ampliación de las capacidades de los estudiantes, y supone, desde la perspectiva de los entrevistados, una contribución importante a la 'dignificación' de la educación, y a la 'motivación y autoestima' de los estudiantes (que han desarrollado un sentimiento de apropiación e integración, contagiado incluso a la comunidad). Pese a los innegables avances, siguen presentes algunas deficiencias, como la falta de docentes especialistas (sobre todo en informática), o insuficiencias del servicio de internet (con la consiguiente infrautilización de los equipamientos); en otras ocasiones el problema es la falta de intervención en el conjunto del centro educativo (en dos de ellos no se había acometido ningún tipo de intervención). 
Figura 3. Valoración de los efectos positivos de las actuaciones en los centros educativos (1)

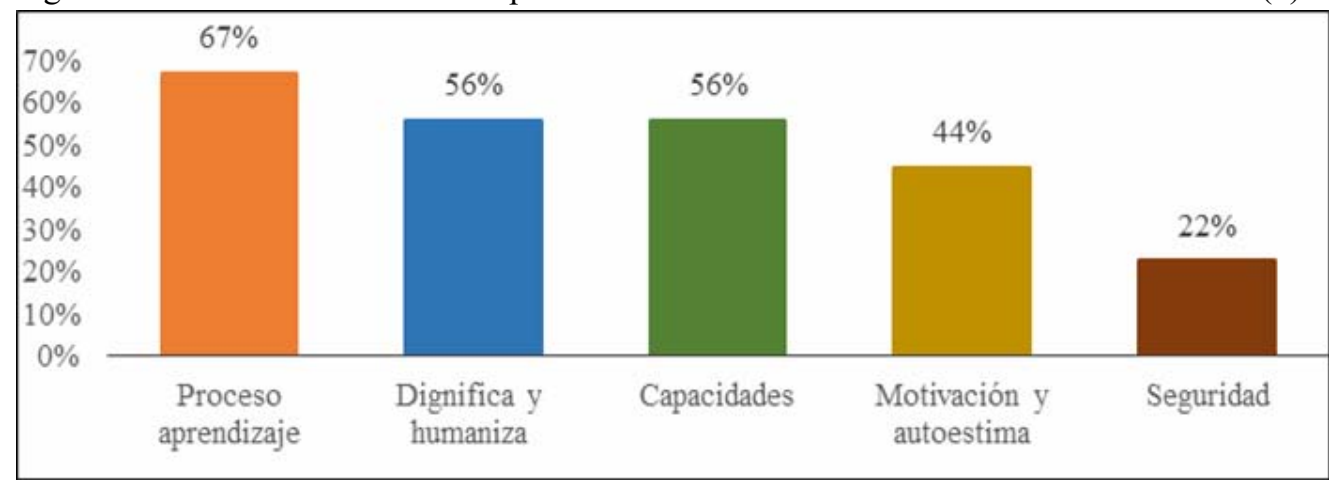

(1): \% sobre el total de actores que abordan el tema educativo.

Fuente: Elaborado a partir de las entrevistas realizadas a los actores.

Figura 4. Escuela Municipal Tierras Coloradas, tras las obras de mejora (Loja, Ecuador).

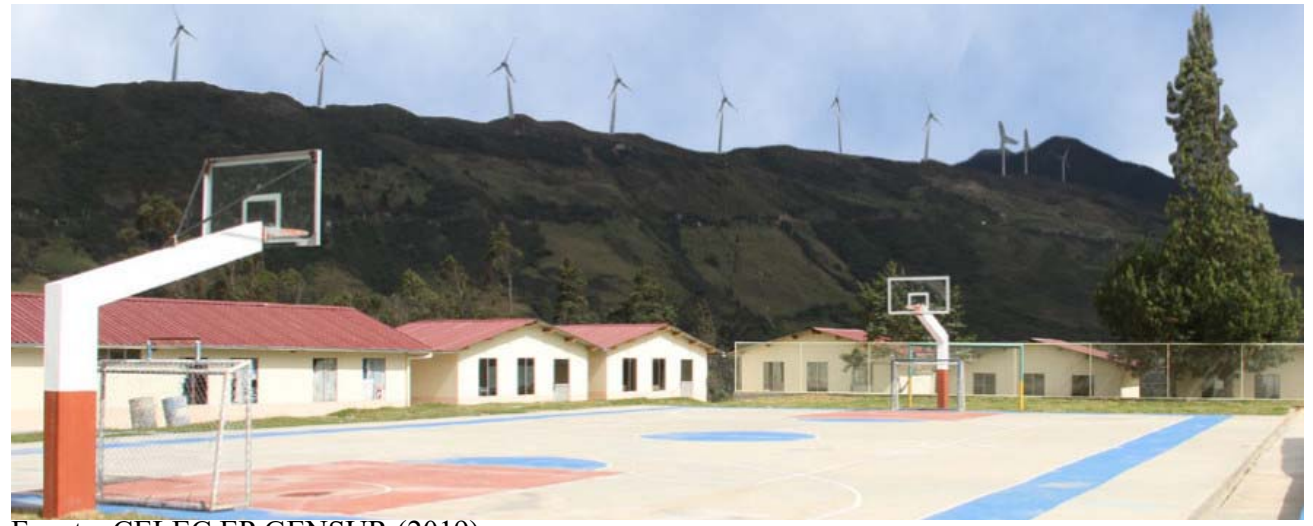

Fuente: CELEC EP GENSUR (2019)

En segundo lugar, otro importante ámbito se refiere a las infraestructuras viarias y sistema de saneamiento. Como parte del PDTC se incluye todo un conjunto de infraestructuras (y de alcantarillado asociado), ejecutadas a través de convenios de colaboración con entidades públicas locales (GAD Municipal de Loja) o nacionales (Ministerio de Transporte y Obras Públicas) (Cuadro 2). Si bien se trata, principalmente, de inversiones productivas destinadas a facilitar la construcción y posterior operación del PEV, diferentes actores ponen el acento en la mejora de la accesibilidad hacia y desde los centros urbanos próximos (Figura 5) y en la reducción de costes de transporte, con efectos claramente beneficiosos para la comunidad local. Entre estos, destaca el impulso a la comercialización de la producción agrícola, o el aumento de visitantes (especialmente al parque eólico y a su centro de interpretación, al ser una novedad para la población ecuatoriana en estos primeros años). Sin embargo, el efecto mejor valorado es el relativo al transporte público. Efectivamente, 
las actuaciones en infraestructuras viarias han tenido efectos positivos en ámbitos tan diversos como el significativo aumento de la oferta de transporte público que, a menor coste, ha permitido mejorar la conexión con el centro urbano de Loja (reduciendo así la sensación de aislamiento y la tradicional dependencia del vehículo privado). A su vez, la proximidad de las infraestructuras viarias ha contribuido igualmente a la revalorización de terrenos, así como a aspectos aparentemente tan poco llamativos como mejoras en la seguridad de los viajeros (al poder atravesar de manera más segura áreas conflictivas en términos de delincuencia).

Pese a los avances, los actores destacan igualmente aspectos que deben ser abordados y mejorados con cierta urgencia, como el mantenimiento de las vías (dado que las frecuentes precipitaciones derivan en su deterioro, y ocasionalmente en derrumbes), la seguridad de los peatones (debido a que la mayor parte de las vías carecen de arcén) y, sobre todo, las redes de alcantarillado (con importantes defectos de funcionamiento, además de contribuir a deslizamientos de tierras que han afectado a viviendas y parcelas adyacentes).

Figura 5. Valoración de los efectos positivos de las mejoras en las infraestructuras viarias (1)

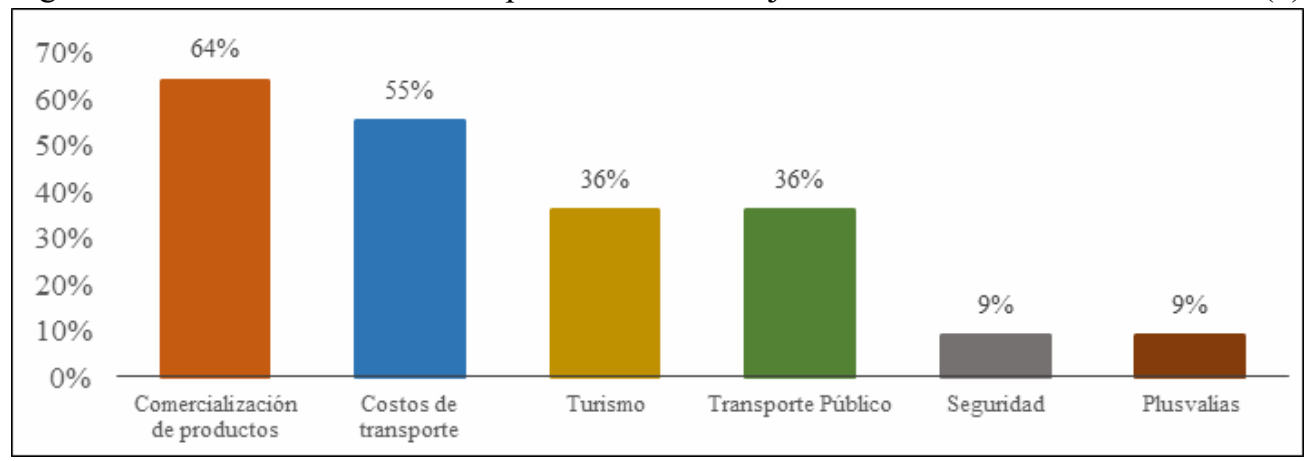

(1): En \% de actores que destacan tema de infraestructuras viarias.

Fuente: Elaborado a partir de las entrevistas realizadas a los actores.

En tercer lugar, respecto a la electrificación, el diagnóstico de necesidades de servicios básicos había evidenciado deficiencias en la calidad del suministro eléctrico y el acceso al servicio de electricidad en la zona de influencia del PEV. Así por ejemplo, en el año 2010 el 18,7 \% de los hogares de la zona urbana del cantón Loja carecían de conexión a la red eléctrica (INEC, 2010). Para abordar estas deficiencias el PDTC incluye todo un conjunto de obras de electrificación para seis barrios del área de influencia (Cuadro 2). El acceso a la electricidad implica, en primer lugar, cambios importantes en las condiciones de vida de la población, con suministro de alumbrado público, que a su vez contribuye a mejorar la imagen de los barrios, a la movilidad de la población, a una mayor seguridad en zonas conflictivas, o a disponibilidad de horarios más amplios para la realización de actividades diversas (recreativas, deportivas, religiosas, cívicas, etc.). Todo ello puede contribuir a una mayor vida comunitaria y, en definitiva, al fortalecimiento del capital social del área 
(Figura 6). Además, en segundo lugar, un mayor y mejor suministro eléctrico constituye también una oportunidad para nuevos emprendimientos que requieren el uso de equipos eléctricos, contribuyendo a la creación de puestos de trabajo y a la mejora de ingresos de las familias (Mendieta, D. y Esparcia, J., 2018).

Figura 6. Alumbrado público en Obrapía, tras las mejoras (Loja, Ecuador).

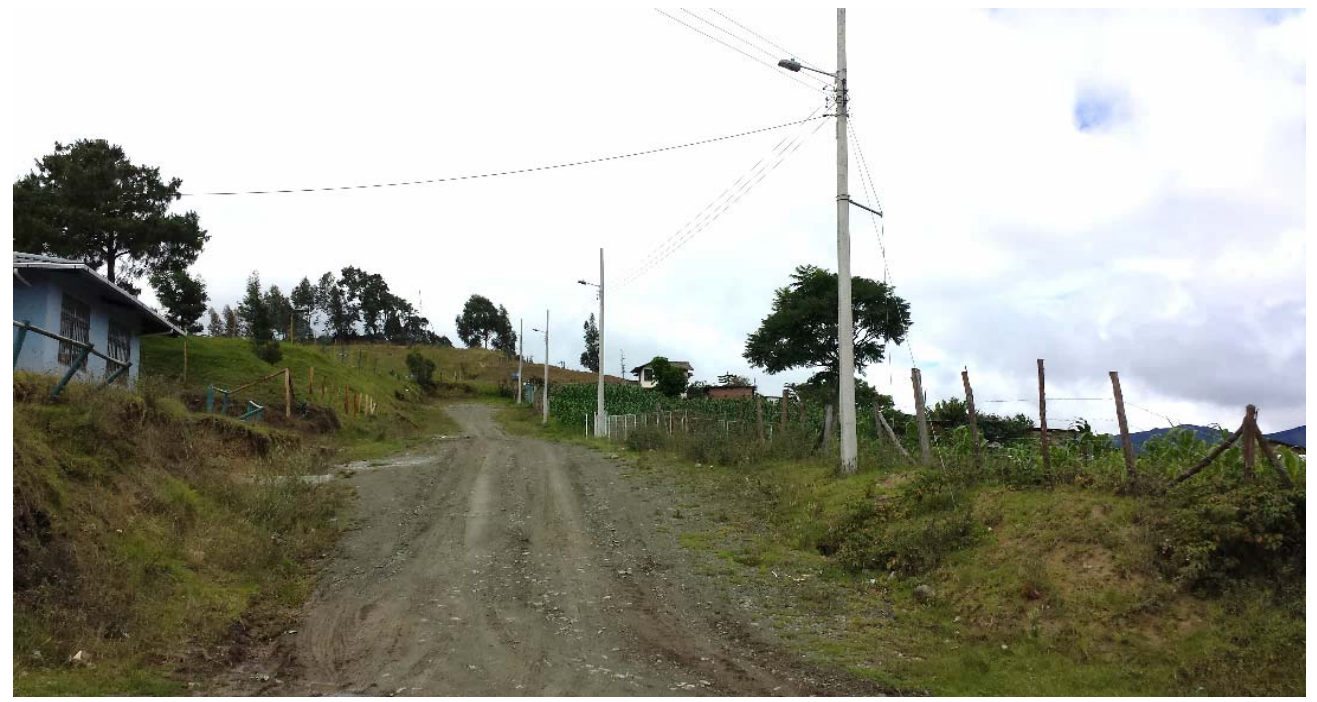

Fuente: CELEC EP GENSUR.

Las importantes mejoras en seis de los barrios no ocultan tareas pendientes, bien en otros sectores de los mismos, o bien en otros barrios, en los que o no se han llevado a cabo actuaciones de electrificación, o estas son insuficientes. En este sentido, resulta paradójico que en zonas muy próximas al parque eólico haya aún una dotación insuficiente, con hogares sin acceso al servicio, o en los que la calidad de este es muy deficiente (como ocurre en la zona oeste del cerro Villonaco).

\subsection{Efectos sobre la creación de empleo}

Los efectos sobre el empleo directo han sido limitados. Estos varían entre la fase de construcción y la de operación del PEV (Figura 6). Así, en línea con lo que se produce en otras plantas de energías renovables (Osti, 2016), en la primera fase se tiende a contratar más mano de obra y con contratos más cortos (174 trabajadores de promedio en la primera y solo 37 en la segunda). No obstante, aunque las necesidades de cualificación son elevadas en ambas fases, es en la segunda fase, de operación y mantenimiento, donde hay una mayor concentración de mano de obra cualificada (67 $\%$ frente al $56 \%$ de la fase de construcción) (Figura 7). La reducida cualificación de la mano de obra local explica que ésta haya constituido el grueso de la contratación 
durante la fase de construcción, pero igualmente que a medida que se ha reducido la contratación y ha aumentado la necesidad de cualificación, el peso de la mano de obra local se haya reducido significativamente (hasta suponer apenas una cuarta parte del total de trabajadores en la fase de mantenimiento).

Figura 7. Empleo generado por el Parque Eólico Villonaco (1)

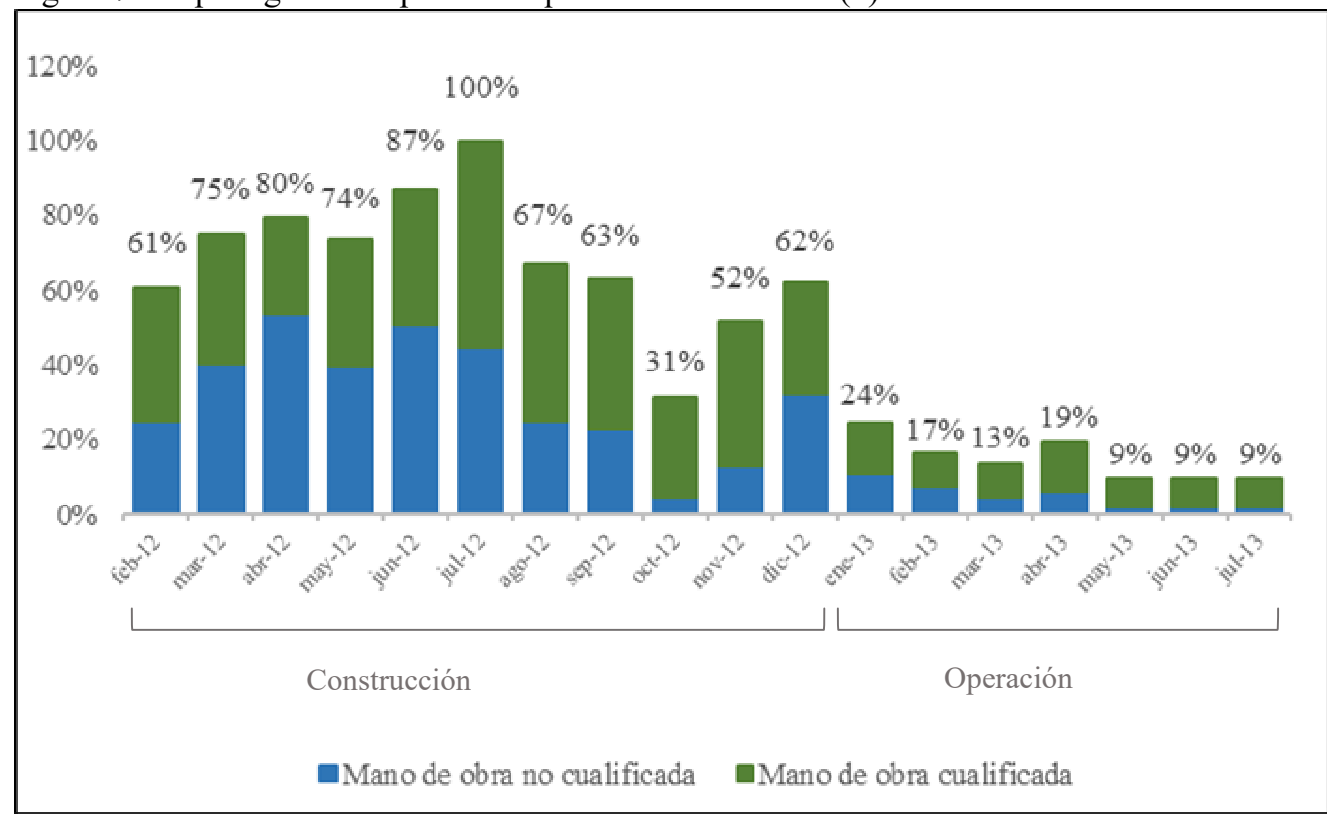

(1) Datos como porcentaje del empleo máximo alcanzado (254 empleos, julio de 2012)002E

* Sin datos para el primer semestre de construcción (agosto 2011- enero 2012).

Fuente: Elaborado a partir de MEER (2015).

Pese a los limitados efectos directos sobre el empleo local, la puesta en marcha del PEV constituye un factor de cierta dinamización del empleo local indirecto, bien bajo la forma de servicios complementarios o bien como empleo temporal vinculado a las obras del PDTC (Cuadro 3). Respecto a los servicios complementarios, los actores entrevistados coinciden en resaltar el papel de la propia empresa de generación al impulsar la creación de una asociación participada por población local, centrada en la prestación de servicios de limpieza, jardinería, mantenimiento de áreas verdes y cafetería en el centro de interpretación. Aunque el nivel de cualificación requerido en este tipo de tareas es, por lo general, bajo, ha sido necesario desarrollar algunas competencias básicas, a lo cual también han contribuido otras entidades (como el Instituto de Economía Popular y Solidaria y el Ministerio de Turismo). Como resultado, se han creado en torno a 50 empleos adicionales, con base local y, lo más importante, con cierta estabilidad, teniendo en cuenta el horizonte de operación de la Central (en torno a 20 años). Este empleo también cuenta con el apoyo preferencial 
previsto en la Ley de Contratación Pública (2008), cuyo objetivo es el de favorecer a los actores de la economía popular y solidaria de la zona donde se ejecuta el contrato.

Cuadro 3. Tipo de empleo creado como efecto de la presencia del PEV en el territorio

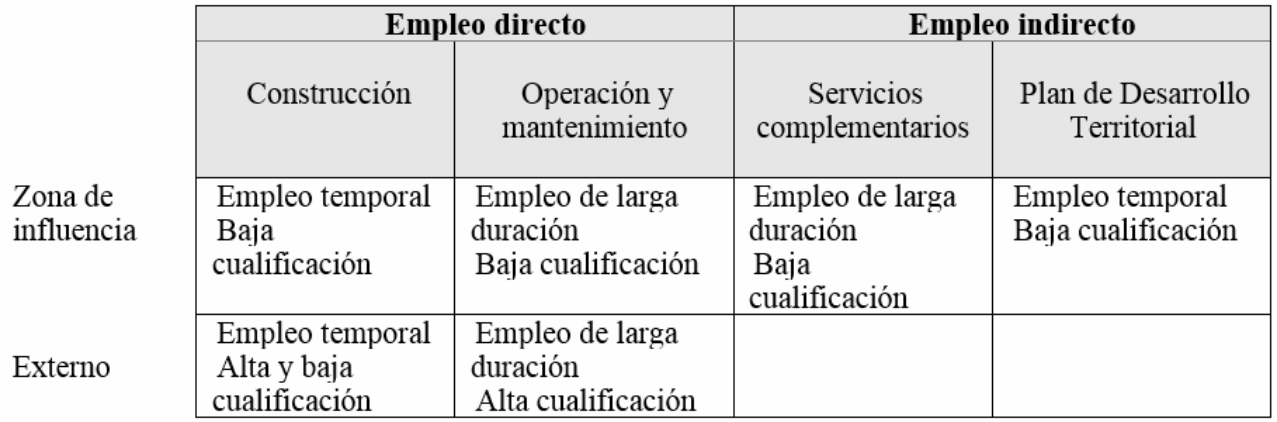

Fuente: Elaborado a partir de los datos recopilados en el trabajo de campo.

Por su parte, las actuaciones del PDTC han tenido efectos mucho más limitados sobre la dinamización del empleo local indirecto, centrados en las obras de construcción de los centros educativos y las de infraestructura vial. Por tanto, se ha tratado principalmente de mano de obra poco cualificada. En el ámbito de la distribución de electricidad la movilización del empleo local ha sido nula, debido a que la empresa de distribución dispone de su propio personal (que cuenta con la cualificación necesaria).

\subsection{Efectos sobre las actividades económicas del territorio}

Dos son las principales posibles fuentes de dinamización de instalaciones como la del PEV, y que en parte recoge el propio PDTC. Por un lado, la atracción de visitantes, lo cual supondría, según las previsiones, un estímulo a actividades de servicios. Por otro, la demanda de bienes y servicios especializados destinados al funcionamiento y mantenimiento de PEV, que igualmente habría de estimular el tejido productivo local.

Respecto de la primera cuestión, la atracción de visitantes, en la literatura internacional se subrayan, por un lado, los efectos negativos de los parques eólicos, especialmente con relación al turismo de naturaleza (Frolova y Pérez, 2008; Molnarova et al. 2012; Kaygusuz et al., 2018). Sin embargo, por otro lado, desde la corriente del energy tourism (Frantál y Urbánková, 2014) se pone de relieve el atractivo que, en diversas ocasiones, pueden tener los propios parques y sus centros de interpretación asociados (Burguillo y del Río, 2008; Frantál y Kunc, 2011), en la medida en la que este tipo de instalaciones también dan lugar a un nuevo paisaje rural (de Andrés Ruiz e Iranzo García, 2011; Morales y Herrero, 2013). De los datos aportados por los entrevistados se desprende que durante los primeros años el PEV y su centro de interpretación están atrayendo una media de unas 3.000 visitas mensuales (en línea con las previsiones del PDTC); sin embargo, la clave aquí es en qué medida 
esas visitas están suponiendo una dinamización significativa de actividades locales. Los datos ponen de relieve que los efectos son, por el momento, escasos, casi limitados a la puesta en marcha de una cafetería en el centro de interpretación.

En cuanto a la demanda de bienes y servicios especializados desde el PEV, es cierto que, como se señala en la bibliografía, con frecuencia determinadas instalaciones industriales o productivas dan lugar a efectos derivados, no solo sobre el empleo, sino que también tienen efectos multiplicadores sobre otras actividades económicas en el territorio (Méndez, 2006). La cuestión es donde se localizan estos potenciales efectos multiplicadores porque no siempre dinamizan al tejido empresarial local o más próximo (Munday et al., 2011). Este es precisamente el caso del PEV, puesto que las entrevistas a actores locales revelan que los principales efectos multiplicadores se han localizado fuera del área de influencia directa del PEV, debido, sobre todo, a la debilidad del tejido productivo local, incapaz de responder a las necesidades derivadas de la nueva actividad.

En general los actores locales son conscientes de los limitados efectos multiplicadores que tiene el PEV, y por ello plantean una serie de propuestas orientadas a hacer de este un factor del desarrollo económico local. En concreto, consideran que es necesario poner en valor el patrimonio natural, cultural y gastronómico del territorio, y generar y mejorar una adecuada oferta de servicios turísticos (Cuadro 4). En este contexto hay que situar la respuesta colectiva de la comunidad local, con la creación y puesta en marcha de una asociación a través de la cual se están canalizando diferentes esfuerzos, principalmente orientados a la prestación de diversos servicios a las instalaciones del PEV (entre ellos el de limpieza-jardinería en el PEV y cafetería-restaurante en el centro de interpretación).

Cuadro 4. Propuestas de los actores para potenciar el desarrollo turístico de la zona

\begin{tabular}{|l|l|l|l|}
\hline \multicolumn{2}{|c|}{ Actores } & \multicolumn{1}{c|}{$\begin{array}{c}\text { Tipo de } \\
\text { aprovechamiento }\end{array}$} & Propuesta \\
\hline Económicos & Agricultores & $\begin{array}{l}\text { Turismo de } \\
\text { naturaleza y } \\
\text { deportivo }\end{array}$ & Puesta en valor del patrimonio natural \\
\hline Institucionales & $\begin{array}{l}\text { GAD Municipal } \\
\text { de Loja }\end{array}$ & $\begin{array}{l}\text { Turismo religioso y } \\
\text { cultural }\end{array}$ & $\begin{array}{l}\text { Puesta en valor del patrimonio } \\
\text { religioso y cultural (iglesias, capillas, } \\
\text { fiestas populares, etc.) }\end{array}$ \\
\cline { 2 - 4 } & $\begin{array}{l}\text { CELEC EP } \\
\text { GENSUR }\end{array}$ & Turismo recreativo & $\begin{array}{l}\text { Construcción de un complejo turístico } \\
\text { recreativo (piscifactoría y pesca } \\
\text { deportiva) }\end{array}$ \\
\hline Sociales & $\begin{array}{l}\text { Presidentes de } \\
\text { barrios }\end{array}$ & $\begin{array}{l}\text { Turismo } \\
\text { gastronómico y } \\
\text { comunitario }\end{array}$ & $\begin{array}{l}\text { Puesta en valor de la gastronomía } \\
\text { típica local }\end{array}$ \\
\hline
\end{tabular}

Fuente: Elaborado a partir de las entrevistas realizadas a los actores. 
Más allá del compromiso desde el PDTC de apoyar las iniciativas que pudieran surgir en cuanto a prestación de servicios (limitadas a actividades de baja cualificación), se contemplaban acciones de apoyo al sector agropecuario, mediante acuerdos con otras entidades. Destacan las acciones formativas (para que los productores puedan mejorar las condiciones de producción); acciones de capacitación preventiva de los productores locales (aunque estas actuaciones se han llevado a cabo solo en cinco de los siete barrios de la zona de influencia); acciones relacionadas con la vigilancia fitosanitaria y zoosanitaria (realización de un diagnóstico de las plagas y enfermedades que afectan a las producciones en el territorio); y, por último y especialmente, en colaboración con el Ministerio de Agricultura y Ganadería (MAG), se había previsto un amplio proyecto de apoyo al desarrollo productivo (que, pese a las previsiones, no ha tenido avances significativos).

\section{Discusión}

Los resultados que se han presentado constituyen un ejemplo de la particularidad de la política energética del estado ecuatoriano (impensable si las empresas generadoras tuviesen carácter privado) que, además de sus objetivos sectoriales (muy modestos, dado que aporta solo el 0,21 \% de la energía del país, y el 0,35\% de las renovables MEER, 2018-), pretende fomentar procesos de desarrollo local y contribuir a mejorar las condiciones de vida de la población local. Estos otros objetivos han sido utilizados por el estado para legitimar (apoyándose en campañas informativas) y obtener mayor apoyo social para este tipo de proyectos eléctricos (denominados emblemáticos). En el caso que nos ocupa, por ejemplo, el respaldo fue mayoritario (el $89 \%$ de los actores sociales, económicos e institucionales respaldaron el proyecto, y el resto tenían solo una posición indiferente o neutral).

Este apoyo ha sido esencial para el mantenimiento del diálogo entre la empresa de generación y los representantes de las asociaciones de vecinos de cada barrio. Un ejemplo de esta cooperación fue el diagnóstico de necesidades, que sirvió de base para el diseño del PDTC, en el cual hubo una destacada participación de la población local. La población local es consciente de las insuficiencias, como la casi nula participación en la priorización de actuaciones, o los resultados, en cuanto a la ejecución de estas, aun limitados. No obstante, se muestran esperanzados en que a medio plazo todo ello pueda contribuir, de forma cada vez más significativa, tanto a la calidad de vida de la población como a la dinamización de las actividades productivas en el área de influencia y, en definitiva, al desarrollo local (Alburquerque, 2017).

La actitud mayoritariamente favorable de la población se apoya, adicionalmente, en otros dos aspectos. En primer lugar, en la idea de energía limpia, que la empresa se ha encargado de difundir, incluso obviando posibles impactos negativos del PEV que, en todo caso, suelen ser menores que en otras energías renovables (Shamsuzzohaa et al., 2012). En segundo lugar, en el hecho de que la mayor parte de la zona de influencia (donde también se concentran las inversiones y la población) está alejada 
de los aerogeneradores. Sin embargo, en las zonas más próximas, algo más de un tercio de los actores sí han tomado conciencia de algunos efectos negativos (ruido de las turbinas, o afectación a terrenos y vías secundarias provocada por la inestabilidad de los taludes).

De los resultados derivan cuatro grandes ámbitos en los que las actuaciones del PDTC siguen siendo insuficientes, centros educativos (urgente contratación de especialistas, y equipamientos varios en centros en los que no se ha intervenido); infraestructuras viarias (mejora, reparación y mantenimiento); acceso y calidad del suministro eléctrico y alumbrado público; y equipamientos y servicios básicos a la población (suministro de agua potable, alcantarillado). Adicionalmente, el hecho de que los principales déficits afecten sobre todo a los barrios y sectores más pobres de la población, hace que este tipo de obras sigan teniendo la más alta prioridad. Cabe señalar, por tanto, que como pone de relieve la ausencia de una buena parte de las obras y equipamientos públicos previstos, el PDTC está lejos de alcanzar los objetivos específicos planteados.

Este PDTC no es una excepción en el contexto de los programas de compensación a las comunidades locales, derivados de proyectos de energías renovables (Escobar et al., 2016). Las debilidades se podrían explicar, al menos parcialmente, y así lo hacen los entrevistados, desde las competencias que tienen las diferentes instituciones. En este sentido, el rol protagonista de la empresa de generación se inscribe en un modelo de desarrollo local en el que el liderazgo de este nuevo actor reconfigura las relaciones entre los actores del territorio y, por tanto, las dinámicas y procesos locales. Esta posición central la ocupa pese a que no cuenta con las capacidades institucionales ni las competencias legales, pero cuenta con los recursos económicos para sufragar las diferentes inversiones. El poder del nuevo actor es tal que no solo no se articula con los gobiernos locales provincial y municipal, sino que desarrolla una verdadera red clientelar (Grindle, 2016; Borja, 2018) entre los actores sociales y económicos del territorio.

El papel del nuevo actor es tal que, también en el ámbito del empleo (con resultados globales por debajo de los esperados, tanto en el directo como en el indirecto) es el motivador clave de las escasas iniciativas productivas externas. Un ejemplo es el de la asociación surgida, en parte como emprendimiento colectivo para la prestación de servicios cada vez más diversos, pero también derivada de los estímulos de la empresa generadora. Más allá de este papel motivador, es innegable la importancia de esta iniciativa a la hora de poner en marcha la asociación. Esta se ha constituido en un factor tanto de empoderamiento de los socios como de creación de empleo (llegando incluso a superar el empleo actual del propio parque eólico). La esperanza de la comunidad local es que esta iniciativa pueda generar un efecto demostración, y dar lugar a otros emprendimientos, con o sin apoyo de la empresa generadora.

Siendo importantes todos estos procesos, difícilmente el caso que aquí se ha analizado puede considerarse en lo que Vázquez Barquero (2007) denomina 'procesos de desarrollo endógeno'. Resultados similares se encuentran en otros trabajos (Pedroli y Langeveld, 2011; OCDE, 2012; IEA-RETD-TCP, 2016) que 
insisten en que para maximizar los beneficios de los proyectos de energía renovable es necesaria una política de desarrollo adaptada a las particularidades de cada territorio (Tribunal de Cuentas Europeo, 2018; Poggi et al., 2018), así como la aceptación y participación social local en la toma de decisiones (Hernando y Blanco, 2016). En el caso de estudio los resultados ponen de relieve que algunos de los elementos que parecen limitar los procesos de desarrollo son la escasa participación y empoderamiento de los actores locales (principalmente económicos y sociales); la escasa capacidad emprendedora e innovadora del territorio, mecanismo clave para impulsar procesos de desarrollo local (Alburquerque, 2017) y, por último, la elevada dependencia respecto de la empresa generadora.

De cara al futuro parece evidente que se ha aprovechar bien la componente exógena al territorio (Madoery, 2001), porque ese flujo de recursos está limitado al periodo de vida útil del parque élico (en torno a 20 años). Por ello, en el marco del desarrollo local, se ha de procurar avanzar en la diversificación de actividades y el fortalecimiento del tejido productivo local, y todo ello con el protagonismo y empoderamiento de la comunidad local.

\section{Conclusiones}

Desde la perspectiva de los actores locales, los efectos del PEV en su zona de influencia son de varios tipos. En primer lugar, los derivados del PDTC (obras de mejora de infraestructuras y equipamientos), que contribuyen a mejorar la calidad de vida de la población. Sin embargo, los actores ponen el énfasis en los desequilibrios territoriales (con efectos también sociales) en la distribución de las inversiones en el territorio, dado que se han centrado ni en actuaciones de carácter integral, ni tampoco se los sectores de la zona de influencia con mayores necesidades.

En segundo lugar, la reducida capacidad del PEV para crear empleo a nivel local, principalmente temporal y de baja cualificación. El efecto más importante ha sido la creación de una empresa colectiva para la prestación de servicios complementarios, formada por socias trabajadoras de la zona de influencia.

En tercer lugar, ha resultado también limitada la capacidad del PEV para favorecer nuevas actividades económicas o fortalecer las existentes. Por un lado, las actuaciones en el ámbito agropecuario no han contribuido significativamente a reducir los problemas estructurales ni mejorar la posición de los agricultores en la cadena de suministro, con lo que tampoco ha incidido en la mejora de la renta agraria. Por otro lado, tampoco se han cumplido todas las expectativas de que el PEV tuviese un impacto positivo en el turismo, dado que gran parte de los efectos multiplicadores de las visitas se localizan fuera de la zona de influencia.

En cuarto lugar, pese a lo anterior, es cierto que estos modestos procesos de desarrollo local se han beneficiado, a la vez que han estimulado, la organización de la población local (especialmente a nivel de barrios), contribuyendo al desarrollo de las capacidades colectivas y de los liderazgos comunitarios. Sin embargo, ello no se ha traducido en procesos de participación local fuertes, en el empoderamiento local o en 
una movilización amplia de las capacidades locales. Todo ello es condición necesaria (pero no suficiente) en los procesos de desarrollo local con base endógena. Como ponen de relieve diferentes autores, el éxito del enfoque se sustenta y pasa por aprovechar mejor algunos intangibles (además de tangibles como los recursos físicos y humanos del territorio), como esa estructura organizativa y liderazgos comunitarios, capacidades locales y de acción colectiva (Alburquerque, 2017).

De cara al futuro, la zona de influencia del PEV tiene una gran oportunidad derivada de la venta de energía, si se cumple, como señala la LOSPEE (2015), que un $30 \%$ de los beneficios puedan destinarse al desarrollo del territorio. Sin embargo, para potenciar las oportunidades que ofrece la presencia de la central, sería necesario un giro mucho más claro de la política actual hacia el desarrollo local sostenible, integral y de base endógena (Vázquez Barquero, 2007). Este habría de basarse en un mayor protagonismo de los actores locales y la promoción de iniciativas económicas surgidas desde la propia comunidad, poniendo en valor el patrimonio y con una gestión respetuosa con el medio ambiente.

\section{Bibliografía}

ADAS Consulting Ltd.-University of Newcastle (2003): Renewable energy and its impact on rural development and sustainability in the UK. DTI New and Renewable Energy Programme, Newcastle.

Alburquerque, F. (2017): DEL Alburquerque. Desarrollo Económico Local. Material Docente. Recuperado el 9-05-2019 de https://www.delalburquerque.es/

Borja, R. (2018): Enciclopedia de la Política (R. Borja, Editor). Recuperado 23-05-2019, http://www.enciclopediadelapolitica.org

Burguillo, M. y Del Río, P. (2008) : La contribución de las energías renovables al desarrollo rural sostenible de la Unión Europea: pautas teóricas para el análisis empírico. ICE Tribuna de Economía (845), 149-165.

Burrows, L. (2018): The down side to wind power: Wind farms will cause more environmental impact than previously thought. The Harvard Gazette. October 4. En https://news.harvard.edu/gazette/story/2018/10/large-scale-wind-power-has-its-down-side/

CELEC EP GENSUR (2019): Sitio web de CELEC EP GENSUR. Recuperado el 23-05-2019 de https://www.celec.gob.ec/gensur

Creswell, J. (2014): Research Design. Qualitative, Quantitative and Mixed Methods (4a ed.). Thousand Oaks (CA): SAGE Publications.

De Andrés Ruiz, C. e Iranzo García, E. (2011): Desarrollo de las energías renovables y cambios paisajísticos: Propuesta de tipología y localización geográfica de los paisajes energéticos de España. En V. Gozálvez Pérez, y J. A. Marco Molina (Ed.), XXII Congreso de Geógrafos Españoles. Energía y territorio: dinámicas y procesos, (pp 97-107). Alicante.

Dos Santos, T. (2003): La teoría de la dependencia: balance y perspectivas. Plaza Janés, Buenos Aires. 
Escobar, R., Gamio, P. M. y Vásquez, U. (2016): Energización rural mediante el uso de energías renovables para fomentar un desarrollo integral y sostenible. Propuestas para alcanzar el acceso universal a la energía en el Perú. Lima, Cecilia Heraud.

Faulín, J., Lera-López, F., Arizkun, A. y Pintor, J. M. (2009): Energy Policy in renewables and its economic and environmental consequences at regional level: The case of Navarre (Spain). Energy Policy: Economic Effects, Security Asspects and Environmental Issues; Jacobs, NB, Ed, 223-256.

Frantál, B. y Kunk, J. (2011): Wind turbines in tourism landscapes. Annals of Tourism Research, 38 (2), 499-519.

Frantál, B. y Urbánková, R. (2014): Energy tourism: an emerging field of study. Current Issues in Tourism, 20 (13) 1395-1412.

Frolova, M. y Pérez, B. (2008): El desarrollo de las energías renovables y el paisaje: algunas bases para la implementación de la Convención Europea del Paisaje en la Política energética española. Cuadernos Geográficos (43), 289-309.

GAD Municipal de Loja (2011): Plan de Desarrollo y Ordenamiento Territorial del cantón Loja. GAD Municial de Loja.

Grindle, M. (2016): Democracy and Clientelism: How Uneasy a Relationship? Latin American Research Review, 51(3), 241-249.

Hernando, M. y Blanco, G. (2016): Territorio y energías renovables no convencionales: Aprendizajes para la construcción de política pública a partir del caso de Rukatayo Alto, Región de Los Ríos, Chile. Gestión y política pública, 25 (1), 165-202.

Hidalgo, A.L. (2012): Economía Política del Desarrollo y Subdesarrollo. Revisitando la Teoría de la Dependencia. Revista Iberoamericana de Estudios de Desarrollo, (1), 5-27.

INEC (2010): Censo de Población y Vivienda 2010. Quito: Instituto Nacional de Estadísticas y Censos.

IRENA (2017): Renewable energy benefits: Leveraging local capacity for onshore wind. Abu Dhabi: International Renewable Energy Agency.

Kaygusuz, K., Güney, M. S. y Kaygusuz, O. (2018): Renewable energy for rural development in Turkey. Journal of Engineering Research and Applied Science, 7(2), 886-895.

Kvale, S. (2011): Las entrevistas en Investigación Cualitativa. Madrid, Ediciones Morata.

Ley Orgánica del Servicio Público de Energía Eléctrica (2015): Asamblea Nacional. R.O. 418. Quito, Ecuador.

Ley Orgánica del Sistema Nacional de Contratación Pública (2008): Asamblea Nacional. R.O. 395. Quito, Ecuador.

Madoery, O. (2001): El valor de la política en el desarrollo local. En Vázquez Barquero, A. y Madoery, O. (Comp.): Transformaciones globales, instituciones y políticas de desarrollo local. Rosario, Homo Sapiens.

Magnani, N. (2012): The Green Energy Transition. Sustainable Development or Ecological Modernization? Sociologica. Italian Journal of Sociology (2), 1-25.

Markandya, A. (2012): Externalities from electricity generation andrenewable energy. Methodology and application in Europe and Spain. Cuadernos Económicos-ICE, 83, 85100.

MEER (2018): Informe de Rendición de Cuentas 2017. Quito: Ministerio de Electricidad y Energía Renovable. 
Méndez, R. (2006): La construcción de redes locales y los procesos de innovación como estrategias de desarrollo rural. Problemas del desarrollo. Revista latinoamericana de Economía, 37(147), 218-240.

Molnarova, K. S. (2012): Visual preferences for wind turbines: location, numbers and respondent. Applied Energy (92), 269-278.

Morales, E. y Herrero, D. (2013): La contribución de la energía eólica al desarrollo rural en Ampudia. XXIII Congreso de Geógrafos Españoles AGE - Espacios insulares y de frontera, una visión geográfica. (631-640). Palma: AGE y Dept. de Ciències de la Terra, UIB.

Moreno, B. y López A.J. (2008): Las energías renovables: perspectivas e impacto sobre el empleo en Asturias. Revista de Estudios Regionales, 83, 177-195.

Munday, M., Bristow, G. y Cowell, R. (2011): Wind farms in rural areas: How far do community benefits from wind farms represent a local economic development opportunity? Journal of Rural Studies, 27(1), 1-12.

Observatorio Europeo Leader (1999): Fuentes de Energías Renovables, fuentes de desarrollo sostenible. Biblioteca Leader de Desarrollo Rural - Comisión Europea.

OCDE (2012): Linking Renewable Energy to Rural Development. OCDE Green Growth Studies. OECD Publishing.

OIT (2015): Trabajar en el campo en el siglo XXI. Realidad y perspectivas del empleo rural en América Latina y el Caribe. (Panorama Laboral Temático, 3). Lima: OIT / Oficina Regional para América Latina y el Caribe.

ONU (2015): Transformar nuestro mundo: la Agenda 2030 para el Desarrollo Sostenible. Resolución aprobada por la Asamblea General el 25 de septiembre de 2015 A/RES/70/1. Nueva York: ONU.

ONU (2015): Objetivos de Desarrollo Sostenible. Recuperado el 17-054-2019, de https://www.un.org/sustainabledevelopment/es/objetivos-de-desarrollo-sostenible/.

ONU (2010): Resolución 65/151. Año Internacional de la Energía Sostenible para Todos. 1-3. New York.

Osti, G. (2016): Renewables, energy saving and welfare in Italian fragile rural areas. Sociologia e Politiche Sociali, 19(3), 102-118.

Pedroli, B. y Langeveld, H. (2011): Impacts of Renewable Energy on European Farmers Creating Benefits for Farmers and Society. Final Report for the European Commission Directorate-General Agriculture and Rural Development,.

Poggi, F., Firmino, A. y Amado, M. (2018): Planning renewable energy in rural areas: Impacts on occupation and land use. Energy, 155, 630-640.

Poveda, G., Franco, Z., Erazo, E., Ruiz, K., González, J. (2017): Desarrollo local de la nueva matriz energética en el Ecuador desde Coca Codo Sinclair, Revista OIDLES, 22.

Ruiz Ruiz, J. (2009): Análisis sociológico del discurso: métodos y lógicas. Forum: Qualitative Social Reseach, 10(2), Art. 26.

SENPLADES (2009): Plan Nacional para el Buen Vivir 2009-2013: Construyendo un Estado Plurinacional e Intercultural (Segunda ed.). Quito: Secretaría Nacional de Planificación y Desarrollo.

SENPLADES (2013): Plan Nacional para el Buen Vivir, 2013-2017. Quito: Secretaría Nacional de Planificación y Desarrollo. 
Shamsuzzohaa, A., Grant, A. y Clarke, J. (2012): Implementation of renewable energy in Scottish rural area: A social study. Renewable and Sustainable Energy Reviews, 16: 18591.

Slee, B. (2015): Is there a case for community-based equity participation in Scottish on-shore wind energy production? Gaps in evidence and research needs. Renewable and Sustainable Energy Reviews (41), 540-549.

SEFORALL (2019): Sustainable Energy for All. Recuperado de www.seforall.org

Tribunal de Cuentas Europeo (2018). Informe Especial $N^{o} 05$. Energía renovable para un desarrollo rural sostenible: posibles sinergias significativas, pero en su mayoría no materializadas en la práctica. Luxemburgo: TCE.

Vázquez Barquero, A. (2007): Desarrollo endógeno. Teorías y políticas de desarrollo territorial. Investigaciones Regionales (11), 183-210.

Walker, G. y Devine-Wright, P. (2008): Community renewable energy: What should it mean? Energy Policy (36), 497-500.

$\mathrm{Yu}$, B. y Xu, L. (2016): Review of ecological compensation in hydropower development. Renewable and Sustainable Energy Reviews (55), 729-738. 